\section{Los desafíos de la integración regional del MERCOSUR y la cooperación económica y comercial en el nuevo milenio de la realidad global The Challenges of regional integration of MERCOSUR economical and commercial cooperation in the new millennium of global reality}

\begin{abstract}
RESUMEN
Como todos sabemos, la tendencia de la economía, en el mundo actual junto a la revolución científico-tecnológica, unifica espacios y operaciones financieras y comerciales. La integración se debe entender como un espacio cuya finalidad es alcanzar un mayor desarrollo económico y comercial mediante el aumento de productividad, disminución de costos, así como la formación de recursos para el ámbito laboral y de la producción, y la cooperación educativa y cultural; todo esto por medio de la ampliación de mercados, elementos importantes para una política económica y social, tal como lo indica el MERCOSUR (Tratado del Mercado Común del Sur). Es ese contexto, MERCOSUR tiene muchos retos y dificultades que superar y evaluar en su funcionamiento; muchos desafíos en el camino de su integración, además de concertar sus objetivos para lograr un mejor desarrollo de su organización; retos y dificultades que servirán de referencia y reflexión para otros modelos de integración latinoamericana.
\end{abstract}

Palabras claves: Integración y desafíos en el MERCOSUR.

\begin{abstract}
As we all know, the trend of the economy in today's world with the scientific-technological revolution, unifies space and financial and business operations. The integration should be understood as a space whose purpose is to achieve greater economic and trade development through increased productivity, reduced costs, and training resources for the workforce and production, and educational and cultural cooperation all this through the expansion of markets, important elements of economic and social policy, as indicated by the Treaty of the Southern Common Market (MERCOSUR). It is this context, MERCOSUR has many challenges and difficulties to overcome and assess its performance; many challenges in the way of integration, in addition to concert their goals for a better development of your organization; challenges and difficulties serve as a reference and reflection for other models of Latin American integration.
\end{abstract}

Keywords: Integration and challenges in MERCOSUR.

\section{Máximo Alfredo Ugarte Vega Centenos}

mugartev@yahoo.com

Universidad Nacional Mayor de San Marcos, Facultad de Ciencias Administrativas

(C) Los autores. Este artículo es publicado por Gestión en el Tercer Milenio de la Facultad de Ciencias Administrativas de la Universidad Nacional Mayor de San Marcos. Este es un artículo de acceso abierto, distribuido bajo los términos de la licencia Creative Commons Atribucion - No Comercia_Compartir Igual 4.0 Internacional. (http://creativecommons.org/licenses/by-nc-sa/4.0/) que permite el uso no comercial, distribución y reproducción en cualquier medio, siempre que la obra original sea debidamente citada. 


\section{INTRODUCCIÓN}

La integración de América Latina no puede ser analizada separadamente. La historia une a los países del continente donde distintas potencias tuvieron dominio (España, Inglaterra, Estados Unidos), cuyos vínculos han estado entorpecidos por conflictos pasados.

En esa orientación, es de mencionar que la integración regional del MERCOSUR se lleva a cabo en el interior de otra integración que vincula a los países de América Latina con el mercado mundial Igual que el caso de MERCOSUR. En ese contexto, Argentina, Brasil Paraguay y Uruguay empezaron el largo camino de la integración mediante el Acuerdo de Constitución del MERCOSUR.

Los países integrantes del MERCOSUR no han querido estar al margen de la integración Europea que ya había empezado hace unas décadas y la tendencia del resto del mundo de adoptar -en algunos casos el inicio, en otros la profundización de espacios económicos de integración-, con el objetivo de beneficiarse de los niveles de producción y del comercio. Como todo proceso de integración, existen dificultades a superar y evaluar en el funcionamiento, y concordar algunos objetivos para un mejor funcionamiento de la organización. En el caso de MERCOSUR, el proyecto de unión aduanera no funcionó; tampoco se logró la libre circulación de mercaderías y pareciera que todo se gobierna con sistemas que se asemejan al funcionamiento de una zona de libre comercio. En otras palabras, existe consenso por declarar la insatisfacción con un estado actual del proceso integrador, Que se caracteriza por ser una combinación de zona de libre comercio imperfecta y una unión aduanera imperfecta; singularidades integracionistas que se desenvuelven dentro del contexto de una debilidad institucional y una falta de política regente orientada al desarrollo regional, lo que significa superar las diferencias históricas entre los países en la construcción de ciertas instituciones supranacionales de integración, así como la modernización del marco legal y regulatorio.

En efecto, en la actualidad, el Mercado Común del Sur (MERCOSUR) es una unión subregional y, al mismo tiempo, es un acuerdo comercial, una unión aduanera todavía imperfecta y tiene una superficie total de $12^{\prime} 794.689 \mathrm{~km}^{2}$ y su población al censo del 2011 llega a 275'676.000; teniendo una densidad de 21.18 habitantes $/ \mathrm{km}^{2}$, así como un Producto Bruto Interno (PBI o PPA) total al 2013 de U\$S 3.436 .988 (con una estimación de U\$S 3.641.734 al 2014) y PBI per cápita de U\$13.347.

MERCOSUR es considerado el cuarto bloque económico y el mayor productor de alimentos del mundo. Tiene por objetivo una integración política, cultural, así como promover el libre intercambio y movimiento de bienes, servicios, personas y capital entre países. Se creó en 1985 gracias a la iniciativa de Argentina y Brasil, y luego se incorporaron Uruguay (1988), Paraguay (1991) y Venezuela (2012). Como estados asociados se encuentran Chile, Colombia, Ecuador, Perú y Bolivia (este último en proceso de incorporación), y como países observadores se encuentra Nueva Zelanda y México (Arnaud 1996).

Argentina y Brasil fueron encaminando sus compromisos pactados en un comienzo para, posteriormente, firmar nuevos acuerdos y acortar los plazos para alcanzar una mayor integración en términos económicos; mientras que Uruguay se fue incorporando paulatinamente por medio de unos protocolos del Programa de Integración y Cooperación Argentino-Brasileño. En 1990, Argentina y Brasil acordaron establecer un mercado común, al que se incorporaron también Uruguay y Paraguay; este último fue suspendido $\mathrm{y}$ reincorporado el 13 de julio del 2013 en cumplimiento del protocolo de Ushuaia sobre el compromiso democrático. Así pues, en marzo de 1991, los cuatro países suscribieron el Tratado de Asunción para establecer el Mercado Común del Sur. Venezuela solicita su incorporación al MERCOSUR el 04 de julio del 2006 y formaliza su ingreso como país miembro en el año 2012 por la falta de ratificación del protocolo por el Congreso Paraguayo.

\section{Planteamiento del estudio}

Dicho esto, y coincidiendo con diferentes autores, cabría preguntarse: ante las nuevas realidades globales y regionales en la escena mundial ¿es importante generar un espacio económico de reducción arancelaria y un 
crecimiento del comercio internacional entre los países miembros de dicho espacio de integración, si los instrumentos jurídicos institucionales que se vienen empleando no son los más convenientes?

\section{Objetivos}

Analizar los desafíos del Mercosur como proyecto de integración regional y las expectativas que despierta dentro y fuera de América Latina como parte de las nuevas realidades globales y regionales.

\section{Justificación}

Las Nuevas realidades globales exigen buscar nuevos espacios de integración en algunos casos y en otros profundizarlos para no ser marginados de los centro de poder económico, comercial y financiero. Asimismo, los desafíos que se van presentando en esta integración sirvan de reflexión y análisis para los demás procesos de integración latinoamericana.

\section{Hipótesis}

En los espacios de integración regional, si se adecuaran a las realidades globales y regionales los diferentes instrumentos jurídicos institucionales; entonces se evitarían un nuevo marco de desafíos y dificultades de integración económica y comercial del MERCOSUR.

\section{Metas Específicas}

Demostrar que las nuevas realidades exigen buscar y consolidar nuevos espacios de integración económica y jurídica.

Definir que Mercosur no solo es un foro debate, sino una organización con muchas dificultades y limitaciones en su proceso de institucionalización.

\section{Marco Teórico y Discusión}

El marco teórico y la discusión del presente trabajo de investigación se dan principalmente, en los siguientes aspectos:

\section{a. Los espacios de integración regional y global en el nuevo escenario internacional}

En el siglo pasado, fundamentalmente en la segunda mitad, se ha configurado una nueva sociedad internacional que sustituyó a la sociedad mundial que se había generado en toda la primera mitad de ese siglo. Actualmente, estamos asistiendo a un creciente cambio de la sociedad internacional, de sus estructuras y dinámicas; que fueron decisivas en la década de los 80 como consecuencia de los cambios en los sistemas políticos y comerciales, o sistemas de Estado.

Así, se ha pasado a una sociedad internacional con nuevas orientaciones en lo político, militar, económico, científico-técnico, cultural,etc.Esdecir,elsistemadelaunipolaridad (sistema militar) y la multipolaridad (sistema político, económico y cultural), dos procesos que en paralelo han tenido repercusiones en la naturaleza de la sociedad internacional, dando como resultado la unificación en el ámbito político-diplomático, estratégico y principalmente jurídico internacional, económico-comercial y de la comunicación e información. En términos generales, podemos decir que la sociedad internacional es mundial y universal, comprendiendo a todos los Estados y la humanidad en sus diferentes actividades.

Como todos sabemos, la globalización es fruto de tres factores principales que facilitaron este proceso: (los factores tecnológicos, económicos y político institucionales). (Del Arenal 2013: 37) y, como consecuencia de estos, surgen los procesos de globalización, interdependencia creciente y transnacionalizaciónque viene experimentando la sociedad internacional (Mindreau Montero 2001:145 a 147). Todo esto genera su vez, la erosión de las fronteras estatales y el papel del Estado en las relaciones económicas internacionales, así como el desarrollo y reforzamiento de las interdependencias y dependencias en los diferentes ámbitos; ya sea político, económico, cultural, estratégico, tecnológico y comercial, entre otros. En otras palabras, los efectos se han dado en todos los ámbitos y las consecuencias han ocurrido en la naturaleza, en el ejercicio distribución del poder y por supuesto en la estructura de la organización de la sociedad internacional.

Ahora bien, estos cambios que se vienen suscitando en la escena internacional van reforzando, paulatinamente, la creciente 
interdependencia marcada, fundamentalmente, por el protagonismo de los estados en el siglo pasado. Hoy, la interdependencia se caracteriza: a) por la existencia y creciente desarrollo de las relaciones económicas regionales y globales, ante las que los propios estados particulares ejercen escasa influencia; debido a la configuración de nuevas instituciones y organizaciones internacionales que lo ha ido debilitando como actor internacional, y que han ido erosionando las fronteras territoriales de los estados, favoreciendo el protagonismo de los actores transnacionales; b) la interdependencia, en sus diferentes manifestaciones desde lo económico comercial hasta lo cultural, ha generado dependencias de todo tipo; c) la interdependencia ha generado un giro radical en cuanto a seguridad nacional, enfrentándose no solo desde un plano nacional; sino desde un plano compartido y común, obligando a los Estados a cooperar desde distintos ámbitos, y d)la interdependencia que reactivó los mecanismos de integración entre los estados para la toma de decisiones conjuntas en todo lo que sea de interés común para los estados miembros; sin que estos signifique perder la identidad, la modernización y la competitividad de estos países.

Estos cambios han desarrollado aspectos favorables como desfavorables en los distintos ámbitos. Entre los favorables podemos citar: en lo político, el fin de la guerra fría ha generado el desarrollo de las democracias como una única alternativa de desarrollo; y en lo tecnológico, las innovaciones y el intercambio de información que posibilitan analizar otras experiencias, en este caso de integración. De otra parte, entre las condiciones desfavorables podemos citar la persistencia de la pobreza, el desempleo, la desintegración social que debilita a la solidaridad y el surgimiento de nuevos desafíos como consecuencia de la crisis financiera internacional que, de una u otra manera, han tenido repercusiones en los diferentes países a nivel mundial.

En la actualidad, siguen existiendo grandes problemas todavía no resueltos, como el de la paz y de la guerra, la explosión demográfica, el agotamiento de los recursos naturales y la degradación ambiental, la desigual distribución de la riqueza, el hambre, la miseria y la opresión, por mencionar algunos.

Esta evolución internacional de la globalización presenta también consecuencias que no solo afectan a las economías, sino también a las sociedades en el ámbito regional(Ferrer 1996: 10 y ss). La creciente disparidad en la distribución de los ingresos (Montoya 1992: 15 y ss) y la falta de transparencia en las comunicaciones traenparalelamente- tensiones sociales en el ámbito regional. Otra consecuencia es la inmigración poblacional, desde los diferentes países pobres hacia los más desarrollados, en la búsqueda de mejores condiciones de vida; es así que los países receptores se ven beneficiados por mano de obra cualificada sin haber realizado ningún costo en ella, en cuanto a su formación y educación (CEFIR 1995:6 y CELADE 1996:30).

En resumen, todos estos cambios que se viene advirtiendo en la sociedad internacional han ido desarrollando nuevas realidades internacionales y regionales; en consecuencia, MERCOSUR no podía estar al margen de las nuevas interdependencias, como señalamos en este mundo complejo y globalizado que pone sobre la mesa la necesidad de un adecuado marco jurídico institucional, ante los desafíos en el ámbito regional.

\section{b. Resultados y dificultades en el proceso de integración del MERCOSUR}

Todo proceso de integración, entre estados, siempre genera conflictos y desavenencias. No existen procesos de integración regional dentro de la experiencia internacional comparada que no haya estado dominado por períodos de conflictos. MERCOSUR no podía estar al margen de estos problemas yaque, talcomo ocurre en todo proceso de integración, desde su creación hasta la fecha se visualizan aspectos positivos y materias pendientes de resolver.

En el ámbito comercial es indudable que el esquema de instrumentación nos muestra aspectos de crecimiento en las corrientes comerciales como consecuencia de la reducción arancelaria, aunque no en los términos de los objetivos planteados primigeniamente. Es de mencionar que tuvo importancia en su 
momento la apertura comercial unilateral de sus Estados miembros.

En la actualidad, hablar de una sola América Latina es cada vez es más difícil por lo mismo que existe varias dimensiones económicas en las que se puede dividir a la región. Una primera sigue siendo aquella que está influenciada por Estados Unidos y que serían México y América Central; mientras que América del Sur estaría siendo influenciada por China por su demanda exterior y, sobre todo, por el precio de las principales materias primas de exportación (BBVA 2013).

Otra manera de visualizar a América Latina sería en base a su apuesta de integración en la economía y en los mercados financieros internacionales; en ese sentido, tenemos a los países de la Alianza del Pacífico que se han propuesto no solo integrarse sino tener presencia en la economía mundial con una visión hacia Asia. En cambio, los países del MERCOSUR siguen retrasados en la liberalización de su comercio con las principales zonas económicas del mundo (Estados Unidos, Unión Europea y China) y Brasil, el pilar del MERCOSUR, no tiene hasta ahora acuerdo comercial alguno con cualquiera de estas potencias.

En la apuesta de la Alianza del Pacífico se auguran proyectos de mayor crecimiento frente a MERCOSUR. El crecimiento en América Latina está previsto que aumente del 2,2\% en el 2013 a 2,5\% en el 2014; sin embargo, es de remarcar que la heterogeneidad de la región seguirá profundizándose. Así tenemos que la Alianza del Pacífico crecerá un 3,8\% en el 2014, más del doble de MERCOSUR que solo llegará al 1,5\%, y en el 2015 se espera la misma proyección (BBVA 2013)

Si se tienen en consideración las cifras del Banco Interamericano de Desarrollo (BID) sobre el desempeño exportador de América Latina en el año 2013, Venezuela es el país del MERCOSURal que le ha ido peor en sus relaciones internacionales. Las exportaciones del bloque decrecieron en $1 \%$ con respecto al año anterior, lo cual no es una crisis y tampoco es una situación positiva y mucho menos es homogénea para todos los países de ese pacto subregional. Hacia el propio MERCOSUR las exportaciones promedio de los países miembros crecieron en un 6\%, reflejando un mejor desempeño del comercio intrarregional en su búsqueda de avanzar y potenciar este bloque (Arancibia 2014).

Desde su inicio, el proceso de integración estuvo acompañado de un marco ideológico no solo en sus objetivos, sino también en los medios y en las instituciones que conducirían el proceso; priorizando la ejecutividad pragmatismo, dejando de lado instancias más representativas. Este bloque se ha caracterizado por tener principios más comerciales que en la búsqueda de valores e identidad característica que debe tener toda organización supranacional. No se llegaron a consolidar la representación de los pueblos y la deliberación legislativa de un parlamento común o un tribunal de justicia supranacionales, por lo que MERCOSUR se está quedando solo como una declaración de principios por la ineficacia de muchas de sus instituciones. Un ejemplo es el Foro Consultivo Económico, cuya creación ya fue aprobada pero que no llega a consolidarse.

Es de mencionar que la eliminación gradual de restricciones no arancelarias no estuvo seguidadelaeliminacióngradualdelosaranceles, debido a la falta de consolidación del libre comercio y de las condiciones de competencia intra-MERCOSUR. Algo similar ocurrió con el Régimen de Adecuación Final (RAF), la eliminación y armonización de restricciones y medidas no arancelarias tradicionales; la armonización de normas técnicas, sanitarias y fitosanitarias; la eliminación de las políticas públicas que distorsionan la competitividad y compras gubernamentales, así como la defensa del consumidor. Si nos remitimos al perfeccionamiento de la política comercial común, todavía falta la implementación del Arancel Externo Común (AEC), los aspectos aduaneros, el tratamiento de las prácticas desleales y salvaguardias para el comercio provenientes de terceros países, y el tratamiento de los derechos de exportación y de las políticas sectoriales; obstáculos que impidieron alcanzar los objetivos de la libre circulación de bienes de la región sin olvidar, tal como señalamos anteriormente, que la unión aduanera tampoco está perfeccionada. 
Si sumamos a esto las características de cada uno de los países miembros como el tamaño, su historia, los ordenamientos jurídicos nacionales y economías de distinto desarrollo, se dificulta aún más la integración. Si nos referimos a los países integrantes, tenemos tamaños diferentes ya que hay dos países pequeños (Paraguay y Uruguay), dos medianos (Argentina y Venezuela) y uno grande (Brasil). Tres de ellos han dejado de lado sus políticas intervencionistas y Venezuela que va en sentido contrario; en cambio Brasil sostiene ciertas posiciones intervencionistas y nacionalistas.

Ahora bien, es de señalar que si bien en el momento de la firma del tratado los países integrantes habían iniciado un proceso democrático que ayudaba la convergencia de sus principios suscritos en la construcción de un ámbito regional, la voluntad política de cada uno de estos países era diferente, así como sus intereses. Mientras Argentina y Brasil habían empezado a integrarse económicamente desde 1988 por medio del Programa de Integración Comercial Argentina-Brasil (PICAB), Uruguay $\mathrm{y}$ Paraguay se sumaron a este proceso por no tener otra posibilidad y así poder subsistir; en cambio Venezuela se integra bajo cuestionamientos severos por parte de uno de los países miembros (Paraguay) por su política y situación intervencionista de su gobierno; aspectos a tomar en consideración por las repercusiones en lo social y en los distintos intereses que motivaron a cada país a participar de este bloque, que tienen incidencia en la voluntad política que se expresa en la solución de diferencias (el Protocolo de Brasilia, el procedimiento de reclamos ante la Comisión de Comercio MERCOSUR) y en las decisiones e instituciones políticas (El Consejo de Mercado Común, el Grupo de Mercado Común, la Comisión de Comercio, la Comisión Parlamentaria Conjunta, el Foro Consultivo Económico y la Secretaría Administrativa) que no son siempre compatibles.

A finales de los 90 parecía que MERCOSUR había ingresado a un período de estancamiento porsituaciones internas (Argentina y Brasil) y externas debido al nuevo contexto internacional en que nos encontramos, situación que está llevando a que esta organización se aleje del objetivo de conformar un mercado común.
En la actualidad la crisis de algunos de los mercados emergentes -como consecuencia de la crisis financiera internacional- está produciendo una devaluación de la moneda tal como ocurre en Argentina, problemaque está acompañado por un estancamiento de su producto bruto y con altas tasas de desempleo, crónico déficit fiscal, creciente endeudamiento externo $y$, como consecuencia, conflictos sociales quese van agudizando.

Frente a estas situaciones, MERCOSUR no señala un rumbo definido ni debate las acciones a tomar, muchas veces solo queda una declaratoria de intenciones; es decir, da por terminado el proceso, evita profundizar más en el nivel de la integración y deja en libertad a sus socios para negociar unilateralmente sus compromisos de integración como es el caso de Argentina, que en diversas oportunidades ha mostrado interés para negociar unilateralmente su adhesión al Área de Libre Comercio de las Américas (NAFTA, conformada por Canadá, Estados Unidos y México) (Arnaud 1996), actitudes que generan confusión y desconfianza entre socios, fundamentalmente en Brasil.

Otras de las tareas pendientes sería la profundización del proceso de integración; es decir, de la unión aduanera inconclusa para lograr un mercado común en la agricultura y en el sector alimentario, la industria, minería, energía, servicios y comunicaciones, transporte e infraestructura, turismo y la coordinación de políticas macroeconómicas y aspectos financieros. Así mismo, estaría pendiente la armonización en los ordenamientos jurídicos nacionales ya que, como se sabe, la integración genera distintas relaciones jurídicas que no necesariamente se encuentran previstas en los ordenamientos jurídicos tradicionales de cada uno de los países integrantes.

Por las dificultades que viene atravesando MERCOSUR, algunos sectores siguen pensando que fue acaso un proyecto rápido y ambicioso; que nació mientras sus miembros se abrían unilateralmente al mundo y estabilizaban sus economías al mismo tiempo, situación que lo hizo más vulnerable a distintos acontecimientos como los problemas económicos y financieros de Argentina y 
Venezuela, dos socios importantes en la región con los que integra el bloque Mercosur. Asimismo, según los expertos advierten que hay problemas estructurales que ahogan, desde hace cuatro años, al mayor país de América Latina y que todos los países que tienen relaciones comerciales con Brasil van a sufrir el impacto en el 2014 como consecuencia de la recesión económica que viene sufriendo.

En resumen, podemos decir que la integración regional, en los países latinoamericanos, viene siendo un desafío que, en la búsqueda de un desarrollo sostenido y una inserción competitiva internacional, ha motivado que experimenten con distintas opciones desde los años 60. MERCOSUR tiene que priorizar su institucionalización $\mathrm{y}$ esperemos que no siga siendo un intento fallido de estos países, y que se pueda dar una real posibilidad de alcanzar sus objetivos inicialmente propuestos.

Las dificultades descritas podrían superarse si el modelo de integración fuera más participativo e inclusivo, y que potencie su inserción en los procesos globales de modernización. La interdependencia de conceptos de democracia y desarrollo facilitaría retomar los principios iníciales de MERCOSUR, dejando de lado los conceptos mercantilistas, evitando así que la integración se convierta en una amenaza (Bitt Alienta s.f. y Pozzo s.f.).

Por último, podemos decir que ante las presiones en todos los ámbito de los centro del poder y para poder disputar su hegemonía, deben de fortalecerse los procesos de integración en América Latina; para que esta parte del continente no sea marginada del poder, y así tener una mejor inserción en la comunidad internacional. MERCOSUR no podía estar al margen de este pensamiento. Así mismo; es de mencionar que las nuevas realidades globales exigen buscar nuevos espacios de integración en algunos casos, y en otros, profundizarlos para no ser marginados de los centros de poder económico, comercial y financiero.

\section{MÉTODO}

Por las características del trabajo de investigación jurídico social se utilizó el método de derecho comparado en materia de evaluación y el tratamiento legal a su normatividad organizacional. Asimismo, se aplicó el método descriptivo para entender la evolución de este proceso y las dificultades que se fueron encontrando en el camino de la construcción integracional.

\section{RESULTADOS}

Ayudar a comprender y difundir los retos en el tortuoso camino de integración regional, es una tarea inevitable para no ser relegados de los centros de poder económico comercial.

Los retos a asumir son muchos, entre estos se debe priorizar su institucionalización y se espera que no siga siendo un intento fallido de estos países que conforman esta organización regional, y que se pueda dar una real posibilidad de alcanzar sus objetivos inicialmente propuestos.

\section{CONCLUSIONES Y RECOMENDACIONES}

El estudio de las nuevas realidades globales exige nuevos espacios de integración, en algunos casos para que los países no sean marginados de los centros de poder y en otros; para profundizar su integración y tener mayor presencia en la escena internacional.

Existen dificultades a superar en el ámbito interno y externo de la integración en la región Latinoamericana. En lo interno, el camino dificultoso de la institucionalización, y en el externo, las tensiones con otros organismos regionales que se van acentuando cada vez más como las que existen con la Alianza del Pacífico, UNASUR, SELA versus ALBA, y MERCOSUR. La orientación de MERCOSUR era más ideológica que económica; la Alianza del Pacífico es más económica que ideológica, y el ALBA (impulsado por Venezuela) era más ideologizado. Todo esto tenía repercusiones, por ejemplo, en el SELA. Cuando se trataba de elegir al director se tenía que Chile, Colombia, México y Perú tenían una posición, y los países más cercanos a MERCOSUR, tenían otra. En UNASUR. También se sentían las repercusiones cuando la cancillerías de los países integrantes de la Alianza asumían la presidencia pro tempore, ya que se percibía más resistencia al bloque de MERCOSUR. 
En resumen podemos decir que:

- MERCOSUR debería profundizar en su propio proceso de integración para que sea más inclusivo, incorporando elementos de justicia social y de vinculación con las sociedades civiles y sus movimientos sociales.

- Su escasa institucionalidad de política supraestatal no desarrolla la construcción de una soberanía compartida.

- El espacio de integración debería de ampliarse a nivel sudamericano y si se pudiera avanzar unificadamente en las negociaciones relacionadas con la Asociación Latinoamericana de Libre Comercio (ALCA), pensando en un espacio hemisférico de bienestar para todos sus habitantes y no solo en la vigencia irrestricta del libre comercio. Así mismo, se pide que MERCOSUR se abra a más acuerdos para establecer compromisos políticos, comerciales, económicos, financieros y de cooperación con otros bloques de integración.

- Se presenta un nuevo marco de desafíos de integración económica y comercial, como es la inserción de las economías de Latinoamérica en el mundo por diferentes medios, como la generación de comercio, la inversión extranjera y su tecnología u obteniendo financiamiento; para esto se requiere adaptabilidad y flexibilidad en estos procesos de inserción en el sistema internacional, a la vez que es necesario redefinir un conjunto de políticas en los diferentes ámbitos de los bloques de integración. En este caso, es necesario que MERCOSUR vaya tomando conciencia de las ventajas competitivas que reclama el mercado mundial.

- MERCOSUR, como proyecto de integración continental, aun con sus propias dificultades, despierta múltiples expectativas dentro y fuera de América Latina; conocerlo, comprenderlo y aportar a su construcción resulta una tarea ineludible.

- A diferencia de la Unión Europea, la estructura institucional de MERCOSUR es de tipo supranacional. Cabe recordar que MERCOSUR funciona con un conjunto de órganos e instituciones de carácter gubernamental.

- El panorama de la integración de MERCOSUR se presenta heterogéneo entre los estados miembros $\mathrm{y}$, de lograrse, tendrían gran impacto en los otros procesos de integración subregional toda vez que sus procesos de integración económica comercial siguen avanzando. MERCOSUR está enfrentando, parcialmente, un sistema de protección de las áreas no competitivas en un mercado abierto más competitivo.

- Se debe considerar que, para incrementar el comercio regional e intrarregional, es necesario impulsar la integración productiva, identificándose proyectos en los cuales pueden agregarse cadenas de valor con un enfoque regional.

- Los países deben orientar su desarrollo dentro de su mismo entorno; es decir, en la misma región del sur donde ha surgido esta iniciativa y donde están los recursos que garanticen su propio desarrollo.

- Y, por último es de interés regional profundizar un debate sobre cómo encarar el nuevo protagonismo de MERCOSUR y tomar en cuenta sus experiencias adquiridas para otros procesos de reciente integración.

\section{REFERENCIAS BIBLIOGRÁFICAS}

ARANCIBIA, Sergio.

2014 "El Peor desempeño". En: TalCualDigital. com. Visitado el 25 de marzo del 2014. Disponible en: www.TalCualDigital.com.

ARMADA NACIONAL DE LA REPÚBLICA ORIENTAL DEL URUGUAY 
s.f. "MERCOSUR: Mercado Común del Sur". Visitado el 22 de marzo del 2013, 9.20 p.m. Disponible en: <http://www.armada.mil.uy/prena/delea/ pdf/el_mercosur.pdf>.

ARNAUD, Vicente.

1996 MERCOSUR, Unión Europea y NAFTA y los procesos de integración regional. Editorial Aboledo-Perrot, Argentina.

INSTITUTO DE STUDIOS INTERNACIONALES.

1989 América Latina y Europa Occidental en el Umbral del siglo XXI. Serie PNUD/CEPAL. Visitado el 20 de marzo de 2014, 6.15 p.m. Disponible en: www.iei.uchile.cl/documentos/ cv.completo_76150_0_5037.docx

BÁRCENA, Alicia.

2012 "Crisis Internacional desacelera comercio latinoamericano". En: Revista de Mercados \& Tendencias. Edición N65.

BBVA

2013 "Informes del BBVA". Visitado el 10 de noviembre del 2013, 10.15 p. m. Disponible en: htpp://serviciodeestudios.bbva.com/KET/ ket/esp/nav/geográficas/latinoamerica/peru/ histórico/publicaciones/observatorios>.

BITT ALIENTA, Gustavo.

s.f. "El impacto de China en América Latina". Visitado el 22 de marzo del 2014, 10.40 p.m. Disponible en: <http://es.scribd.com/ doc/103588484/El-Impacto-de-China-enAmerica-Latina>.

CAETANO, Gerardo

s.f. "Mercosur. 20 años". Visitado el 15 de marzo del 2014, 9.40 p. m. Disponible en: <http://library. fes.de/pdf-files/bueros/uruguay/07904.pdf, Visitado el 22/13/2013>.

CARRAUD, $M$.

1980 L'intégration des pays andins. Editorial Paría, París.

CENTRO DE FORMACIÓN PARA LA INTEGRACIÓN REGIONAL (CEFIR).

1995 "Seminariosobre la Dimensión Social de la Integración Regional”. CEFIR, Santiago de Chile.

CENTRO DE INFORMACIÓN Y DOCUMENTACIÓN INTERNACIONALES EN BARCELONA (CIDOB).

1998 "Organismesd'integracio a América Latina”. En: Revista CIDOB. N40-41.

CENTRO LATINOAMERICANO DE DEMOGRAFÍA (CELADE).
1996 "Impacto de las tendencias demográficas sobre los sectores sociales en América Latina. Contribución al diseño de políticas y programas". CELADE, Santiago de Chile.

COMISIÓN ECONÓMICA PARA AMÉRICA LATINA Y EL CARIBE (CEPAL).

1992 "Integración regional: desafíos y opciones". En:Revista de Comercio Exterior. Enero 1992. Editorial de la CEPAL, México.

COMISIÓN ECONÓMICA PARA AMÉRICA LATINA Y EL CARIBE (CEPAL).

1992 "Tratado de Asunción del Mercado Común del Cono Sur (MERCOSUR): algunas consideraciones". Doc. LC/R. 1118, del 15 enero de 1992. CEPAL, Santiago de Chile.

DEL ARENAL, Celestino.

2013 "La Nueva Sociedad Mundial y las nuevas realidades internacionales: Un reto para la Teoría y para la política". Visitado el 04 de julio del 2013. Disponible en: <www. mexicodiplomatico/lecturasLa nueva sociedad Mundial Realidades Internacionales>.

FERRER, Aldo.

1996 Historia de la globalización. Orígenes del orden económico mundial. Fondo de Cultura Económica, Buenos Aires. pp. 10 y ss.

GARCÍA, H.

1992 "Tratado de Asunción del Mercado Común del Cono Sur (MERCOSUR): Algunas consideraciones". Documento LC/R.118, del 15 de enero de1992. CEPAL, Santiago de Chile.

-INSTITUTO BOLIVIANO DE COMERCIO EXTERIOR.

1991 MERCOSUR versus Grupo Andino. IBCE, Santa Cruz de la Sierra.

-MERCOSUR.

s.f. a) "MERCOSUR, documentos oficiales". Visitado el 22 de marzo del 2013, 10.30 p. m. Disponible en: <http://www.mercosur.int/>.

- MERCOSUR.s.f. b) "MERCOSUR, Reunión Ordinaria del Grupo Mercado Común". Visitado el 22 de marzo del 2013, 10.30 p. m. Disponible en: <http://www.mercosur.int/innovaportal/ file/5602/1/gmc_2013_acta02_es.pdf>

- MERCOSURs.f. c) Entrada en Enciclopedia Libre. Visitado el 15 de mayo del 2013, 9.23. p. m.. Disponible en: <http//es.wilkipedia. org/ wiki//Mercosur>.

MINDREAU MONTERO, Manuel. 
2001 Introducción a la teoría de las relaciones internacionales. Editorial Centro de Investigaciones de la Universidad del Pacífico, Lima.

\section{- MOYA DOMÍNGUEZ, M.T.}

1983 "Argentina ante el proceso de transición de la integración latinoamericana". En: Anuario Argentino de Derecho Internacional. Vol. I.

- MONTOYA, Silvia.

1992 Asimetrías sociales en el MERCOSUR. Fundación Mediterránea - Fundación Konrad Adenauer, Buenos Aires.

\section{- PARLAMENTO DE MERCOSUR.}

s.f. Parlamento de MERCOSUR. Visitado el 21 de marzo del 2013, 10.12 p. m. Disponible en: <http://www.parlamentodelmercosur.org/>.

- PORLLES TORREJÓN, Nancy.

2007 Derecho de Comercio Internacional. Fondo Editorial UGV, Lima.

- POZZO, Gastón.

s.f. "El Mercosur CNAPP". Visitado el 21 de marzo del 2013, 9.15 p.m. Disponible en: <http:// www.cncs.com.uy/docs/documentos_varios/ El\%20Mercosur.pdf>

- RABELO VERSIANI, Flavio y SANTANA, José Antonio.
1987 Brasil, Argentina-Uruguai: a integraçcäo en debate. Editorial BR. Brasil.

- UGARTE VEGA CENTENO, Máximo Alfredo

1994 "Hacia un Nuevo orden económico mundial". En: Cuadernos Jurídicos. N¹5. Editorial Fontalva, Barcelona.

- VACCHINO, J.M.

1988 "La integración Argentino-Brasileña y las relaciones entre América Latina y Europa. Reflexiones complementarias". En: Síntesis. $\mathrm{N}^{\circ} 4$.

- UNIVERSIDAD DE LA REPÚBLICA - RED ACADÉMICA URUGUAYA

s.f. a) “Tratado de Asunción de 1991. MERCOSUR". Visitado el 15 de enero del 2013, 5.15 p.m. Disponible en: <www.rau.edu.uy/mercosur/ trtasp.htm>.

- UNIVERSIDAD DE LA REPÚBLICA - RED ACADÉMICA URUGUAYA

s.f. b) "Protocolo de OuroPreto". Visitado el 19 de junio del 2013, 7.23 p.m. Disponible en: <www. rau.edu.uy/mercosur/opretosp.htm>.

- Wikipedia.

s.f. "Anexo: Países del Mercosur por PIB", Visitado 20 de mayo del 2014, 7.5 p.m. Disponible en : es Wilkipedia.org/wiki/...anexo:Países_del_ Mercosur_por_PBI. 